\title{
Optical nonlinear response of a single nonlinear dielectric layer sandwiched between two linear dielectric structures
}

\author{
E. Lidorikis \\ Ames Laboratory-USDOE and Department of Physics and Astronomy, Iowa State University, Ames, Iowa 50011 \\ K. Busch \\ Ames Laboratory-USDOE and Department of Physics and Astronomy, Iowa State University, Ames, Iowa 50011 \\ and Institüt für Theorie der Kondensierten Materie, Universität Karlsruhe, D-76128, Karlsruhe, Germany \\ Qiming Li \\ Ames Laboratory-USDOE and Department of Physics and Astronomy, Iowa State University, Ames, Iowa 50011 \\ C. T. Chan \\ Ames Laboratory-USDOE and Department of Physics and Astronomy, Iowa State University, Ames, Iowa 50011 \\ and Department of Physics, The Hong Kong University of Science and Technology, Clear Water Bay, Kowloon, \\ Hong Kong, China \\ C. M. Soukoulis \\ Ames Laboratory-USDOE and Department of Physics and Astronomy, Iowa State University, Ames, Iowa 50011
}

(Received 11 February 1997; revised manuscript received 6 May 1997)

\begin{abstract}
We consider the general problem of electromagnetic wave propagation through a one-dimensional system consisting of a nonlinear medium sandwiched between two linear structures. Special emphasis is given to systems where the latter comprise Bragg reflectors. We obtain an exact expression for the nonlinear response of such dielectric superlattices when the nonlinear impurity is very thin, or in the $\delta$-function limit. We find that both the switching-up and switching-down intensities of the bistable response can be made very low, when the frequency of the incident wave matches that of the impurity mode of the structure. Numerical results for a nonlinear layer of finite width display qualitatively similar behavior, thus confirming the usefulness of the simpler $\delta$-function model. In addition, an analytical solution for the resonance states of an infinitely extended finite-width superlattice with a finite-width nonlinear impurity is presented. [S0163-1829(97)02848-8]
\end{abstract}

\section{INTRODUCTION}

Dielectric materials with an intensity-dependent dielectric constant are well known for their complex response to radiation. Exciting features such as bistability, multistability, optical limiting, etc. have been predicted theoretically ${ }^{1,2}$ and observed experimentally. ${ }^{3-5}$ Promising future applications include optical switches and transistors, pulse shapers, as well as memory elements. Already quite simple structures like the traditional Fabry-Perot etalons ${ }^{3}$ or multilayered structures of alternating nonlinear dielectric materials ${ }^{6}$ exhibit such a behavior. The common characteristic of all nonlinear optical devices is the feedback mechanism, necessary to enhance the nonlinear effects. Crudely speaking, there exist two types of realizations: In the case of a "localized" feedback structure a homogeneous nonlinear medium is placed between two reflectors (mirrors ${ }^{3,7}$ or Braggreflectors $^{8}$ ), while "distributed" feedback mechanisms are realized through a periodic modulation of the linear part of the nonlinear materials' refractive index. ${ }^{1}$ Similar studies have been done for the electronic response in a onedimensional nonlinear lattice, ${ }^{9-11}$ as well as for a linear lattice with nonlinear impurities. ${ }^{12}$

The linear (or low-intensity) properties of the feedback mechanism are important for the nonlinear response of the device. For instance, a distributed feedback structure brings about a photonic band gap, ${ }^{13,14}$ where certain modes are forbidden while others propagate freely. For frequencies inside the transmission band, bistability results from a modulation of transmission by an intensity dependent phase shift. For frequencies inside the stop gap of the linear system, bistability and resonance transmission is achieved via gap soliton formation. ${ }^{15,16}$ However, nonlinear media are usually quite lossy and, thus, it is important to find ways of keeping the amount of nonlinear material small while still retaining sizable nonlinear effects. Furthermore, the successful use of optical switches depends crucially on a low threshold, i.e., low "switching" intensities. The above considerations lead to the question of whether a single nonlinear layer, suitably supplied with a feedback mechanism, may be sufficient for optical switching devices. In this paper we present the results of our investigation of this and several related problems.

The paper is divided into two parts: The first part deals with the general case of a "localized" feedback structure when the nonlinear layer is very thin, or in the $\delta$-function approximation. We will give arguments as to why a system consisting of a nonlinear layer sandwiched between Bragg reflectors may be considered the most efficient "localized" feedback structure, as well as which parameters of the system determine the bistability threshold. We also demonstrate 
the usefulness of the $\delta$-function approximation by comparing with numerical results for a nonlinear layer of finite-width. However, a finite nonlinear layer always exhibits multistability. This is illustrated in the second part of this paper, where we present an analytical solution for the resonance states of an infinite superlattice with a finite-width nonlinear impurity layer. These are modulated band-gap impurity modes and correspond to the the transmission resonances in the multistable input vs output diagram. Finally, a comparison between the analytical solution for the infinite superlattice with numerical results for a finite superlattice shows very good agreement.

\section{VERY THIN NONLINEAR LAYER}

Many features of wave propagation through onedimensional nonlinear structures can most conveniently be investigated and understood within a nonlinear KronigPenney $\delta$-function model. ${ }^{17}$ Although discrepancies with the more realistic finite width models do exist, it has been demonstrated that the $\delta$-function model captures most of the essential features of nonlinear response to radiation. ${ }^{18}$ In this section we will consider a very thin nonlinear layer centered at the origin. The nonlinearity is expressed in first order by an intensity dependent term in the dielectric function $\epsilon(x)$ :

$$
\epsilon(x) \approx \epsilon_{0}\left(1+\lambda|E(x)|^{2}\right) \delta(x) .
$$

Here $\epsilon_{0}$ is defined as the limit $n^{2} d \rightarrow \epsilon_{0}$ for $n \rightarrow \infty, d \rightarrow 0$, of a finite width layer of extent $d$ and index of refraction $n$, and $\epsilon_{0} \lambda$ is the corresponding nonlinear Kerr coefficient.

We will first discuss the problem of a single nonlinear $\delta$ function. More insight is obtained from a general discussion of a $\delta$-function nonlinear layer sandwiched between two linear structures. It will be shown that the equation relating input and output intensities has the same form as for a single nonlinear $\delta$ function except for a parameter renormalization. Also, a very useful phase diagram for the onset of bistability in terms of the linear properties of the system will be derived. From this it follows that the most interesting physics appears when the linear structures on the two sides of the nonlinear layer are identical Bragg reflectors. Assuming the latter to consist of very thin layers, we arrive at a remarkably simple but very rich result for the input-output intensity relation.

\section{A. Single nonlinear $\delta$ function}

We first consider electromagnetic wave propagation through a single $\delta$ function with an intensity dependent dielectric strength as given by Eq. (1). This is schematically depicted in Fig. 1. Our interest lies in the steady state response of the system. Let a plane wave $E_{0} e^{-i k x}$ with wave number $k=\omega / c$ be incident from the right. This gives rise to a reflected wave, $E_{r} e^{i k x}$, as well as to a transmitted wave, $E_{t} e^{-i k x}$. Solving the Maxwell equation with a single nonlinear $\delta$-function layer at $x=0$ will yield a relationship between the incident and transmitted intensity, $Y=\left|E_{0}\right|^{2}$ and $X=\left|E_{t}\right|^{2}$, respectively:

$$
Y=X\left[1+\tau(1+\lambda X)^{2}\right]
$$

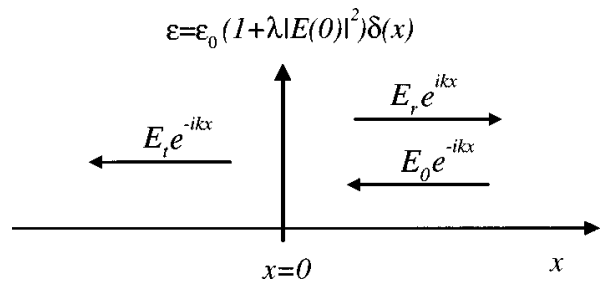

FIG. 1. Geometry considered in this section. A plane wave of amplitude $E_{0}$ strikes a nonlinear $\delta$ function, giving rise to a reflected and a transmitted wave.

where $\tau \equiv \epsilon_{0}^{2} k^{2} / 4$ is related to the transmission coefficient $\left|T_{0}\right|^{2}$ of the corresponding linear $(\lambda=0)$ system, $\tau=\left(1-\left|T_{0}\right|^{2}\right) /\left|T_{0}\right|^{2}$. The system will exhibit bistability if we can have more than one output for a given input. This will be true if $Y$ is a nonmonotonic function of $X$. We find a physical solution for $d Y / d X=0$ only for $\lambda<0$ and $\tau>3$ :

$$
X_{ \pm}=\frac{2 \pm \sqrt{1-3 / \tau}}{3|\lambda|} .
$$

The nonlinear response of such a system with negative $\lambda$ is shown in the input vs output diagram in Fig. 2(a) for various values of $\tau$ and $\lambda$. The absence of bistability for a positive Kerr coefficient is one of the artifacts of the $\delta$-function approximation. We will see later that this restriction is lifted, once the nonlinear $\delta$ function is placed between two linear systems with nonzero reflection coefficients.

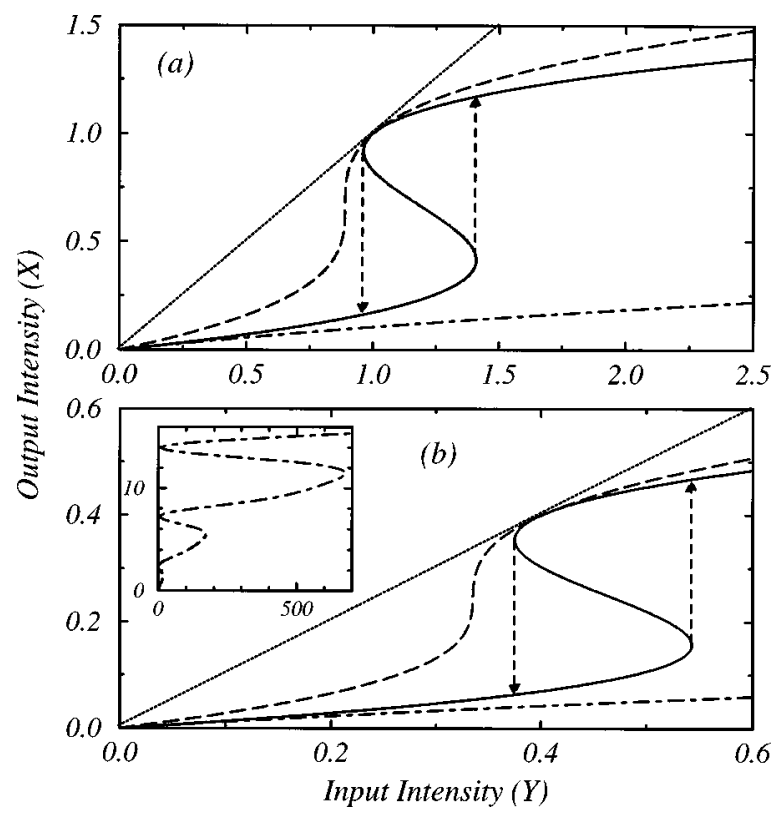

FIG. 2. Transmitted intensity vs incident intensity for (a) a nonlinear $\delta$ function and (b) a nonlinear dielectric slab of width $d=0.6 \lambda_{f}$. In both cases, the dashed line corresponds to $\lambda=-1$ and linear transmission $\left|T_{0}\right|^{2}=0.25$, the solid line to $\lambda=-1$ and linear transmission $\left|T_{0}\right|^{2}=0.125$, and the dot-dashed line to $\lambda=+1$ and linear transmission $\left|T_{0}\right|^{2}=0.125$. Note that the bistability onset happens for the same system parameters. However, for large intensities the finite layer system with $\lambda>0$ will always display multistable behavior [inset graph in (b), for the same parameters: $\lambda=+1$, $\left.\left|T_{0}\right|^{2}=0.125\right]$. The dotted line is the total transmission relation $Y=X$. 
The switching-up and switching-down intensities are given by $Y_{ \pm}=Y\left(X_{\mp}\right)$, respectively. For $\tau=3$ and $\lambda<0$ we have the onset of bistability at $Y_{+}=Y_{-}=8 /(9|\lambda|)$. As $\tau$ gets larger, $\quad Y_{-} \rightarrow 1 /|\lambda|$ while $Y_{+} \sim \tau /|\lambda|$. Note that for $Y=X=1 /|\lambda|$ we have resonance transmission. Thus for large $\tau$ (or equivelantly for low linear transmission) the switchingdown intensity and the resonance intensity are the same. Clearly, the larger $|\lambda|$ is, the smaller the switching intensities are. Note further, that the resonance transmission intensities $Y=X=1 /|\lambda|$ correspond to $\epsilon(0)=0$, i.e., the index mismatch between the $\delta$ function and the background effectively disappears, leading to total transmission. On the other hand, for a finite-width linear layer with dielectric constant $\epsilon=n^{2}$, the condition for resonance transmission is $2 d=m \lambda_{f}$, where $d$ is the width of the layer and $m \lambda_{f}$ is an integer multiple of the wavelength inside the dielectric material $\left(\lambda_{f}=2 \pi c / n \omega\right){ }^{6}$ If we incorporate a nonlinear coefficient in the dielectric constant $\left[\epsilon(x)=n^{2}\left(1+\lambda|E(x)|^{2}\right)\right]$, then for suitable choices of the parameters we can have bistability (and even multistability) for both negative and positive Kerr medium. For example, if $2 d<\lambda_{f}$ and $\lambda>0$, then effectively the dielectric constant will get larger, yielding a smaller average effective wavelength $\left\langle\lambda_{f}\right\rangle$. For some intensity we should expect the resonance condition to be "effectively" satisfied, and, as a consequence, get a transmission resonance. Higher-order resonances are also expected giving rise to multistability. For a negative Kerr coefficient and $2 d<\lambda_{f}$ we should not expect bistability, as correctly pointed out by Chen and Mills, ${ }^{2}$ unless we allow the unphysical situation at which the intensities become large enough to make $\left\langle n_{\text {eff }}^{2}\right\rangle \leqslant 1$. Only then we would get bistability, and this would be the exact correspondence to the $\delta$-function case. For a positive Kerr coefficient, however, bistability should always be expected (although some times only at unrealistically high intensities), a feature that is absent in the $\delta$-function model.

In Fig. 2(b) we show the transmission diagram for a finite-width dielectric slab for $d=0.6 \lambda_{f}$ and various values of linear transmission coefficient and nonlinear Kerr coefficient. Although this is not the exact analog of Fig. 2(a), it is remarkable that both models show a similar dependence on their linear transmission properties. We also find that for larger intensities the negative nonlinear medium does not exhibit multistability, while a positive nonlinear medium will always exhibit multistability, as can be seen in the inset graph in Fig. 2(b).

The unphysical condition for resonance transmission in the single nonlinear $\delta$-function case can be lifted if we sandwich the $\delta$ function between two linear systems. Bistability will then occur as a result of the intensity dependent phase shift that the $\delta$ function will introduce between the two linear systems, which will alter their transmission characteristics. As we will see in the next section, this arrangement is also optimal for obtaining lower switching intensities.

\section{B. Nonlinear $\delta$ function sandwiched between two linear systems}

Let us now consider a more general geometry as shown in Fig. 3, where a nonlinear $\delta$ function is sandwiched between two linear systems, characterized by the reflection and transmission amplitudes, $r_{i}$ and $t_{i}(i=1,2)$, respectively. Gener-

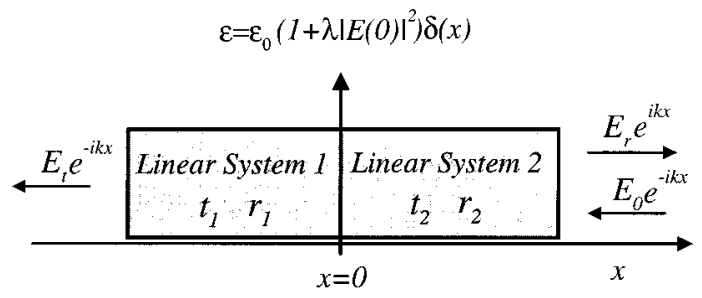

FIG. 3. The general geometry considered in this section. A nonlinear $\delta$ function is sandwiched between two general linear systems characterized by the reflection and transmission amplitudes $r_{i}$ and $t_{i}, i=1,2$. A plane wave of amplitude $E_{0}$ incident upon the system from the right results in a transmission amplitude $E_{t}$ and a reflection amplitude $E_{r}$.

ally, for a linear system, with incident and reflected plane waves from it's left $A e^{i k x}$ and $B e^{-i k x}$, respectively, and incident and reflected plane waves from its right $D e^{-i k x}$ and $C e^{i k x}$, respectively, the relation between the field amplitudes is described by the transmission matrix:

$$
\left(\begin{array}{l}
C \\
D
\end{array}\right)=\left(\begin{array}{cc}
1 / t^{*} & -r / t \\
-(r / t)^{*} & 1 / t
\end{array}\right)\left(\begin{array}{l}
A \\
B
\end{array}\right) .
$$

Just as before, we assume this composed object to be illuminated from the right by a plane wave with amplitude $E_{0}$. The corresponding reflected and transmitted wave amplitudes are denoted by $E_{r}$ and $E_{t}$, respectively. Then, the relation between the output intensity $X=\left|E_{t}\right|^{2}$ and the input intensity $Y=\left|E_{0}\right|^{2}$ can be obtained straightforwardly by properly matching the values of the fields at the origin:

$$
Y=X\left[\gamma+\tau^{\prime}\left(1+\lambda^{\prime} X\right)^{2}\right]
$$

Note that Eq. (5) has exactly the same structure as Eq. (2). Thus, we have shown that the introduction of the linear structures leads to a renormalization of the quantities in Eq. (2): $1 \rightarrow \gamma, \tau \rightarrow \tau^{\prime}$, and $\lambda \rightarrow \lambda^{\prime}$. The quantities $\gamma, \tau^{\prime}$, and $\lambda^{\prime}$ are given by the following expressions:

$$
\begin{gathered}
\gamma=\frac{1 /\left(|T|^{2}\left|T_{0}\right|^{2}\right)-\operatorname{Re}^{2}\left[1 /\left(T^{*} T_{0}\right)\right]}{\left|1 / T_{0}-1 / T\right|^{2}}, \\
\tau^{\prime}=\frac{\left\{1 /\left|T_{0}\right|^{2}-\operatorname{Re}\left[1 /\left(T^{*} T_{0}\right)\right]\right\}^{2}}{\left|1 / T_{0}-1 / T\right|^{2}}, \\
\lambda^{\prime}=\lambda\left|1 / t_{1}-r_{1}^{*} / t_{1}^{*}\right|^{2}\left|1 / T_{0}-1 / T\right| / \sqrt{\tau^{\prime}},
\end{gathered}
$$

where $T$ is the linear transmission amplitude for the system without the $\delta$ function at the origin $\left(\epsilon_{0} \equiv 0\right)$ :

$$
\frac{1}{T}=\frac{1}{t_{1} t_{2}}+\frac{r_{1}^{*} r_{2}}{t_{1}^{*} t_{2}}
$$

Similarly, $T_{0}$ is the linear transmission amplitude for the case with the $\delta$ function at the origin $(\lambda \equiv 0)$,

$$
\frac{1}{T_{0}}=\frac{1}{T}-i \frac{k \epsilon_{0}}{2}\left(\frac{1}{t_{1}}-\frac{r_{1}^{*}}{t_{1}^{*}}\right)\left(\frac{1}{t_{2}}+\frac{r_{2}}{t_{2}}\right) .
$$

The conditions for bistability from Eq. (5) now read as 


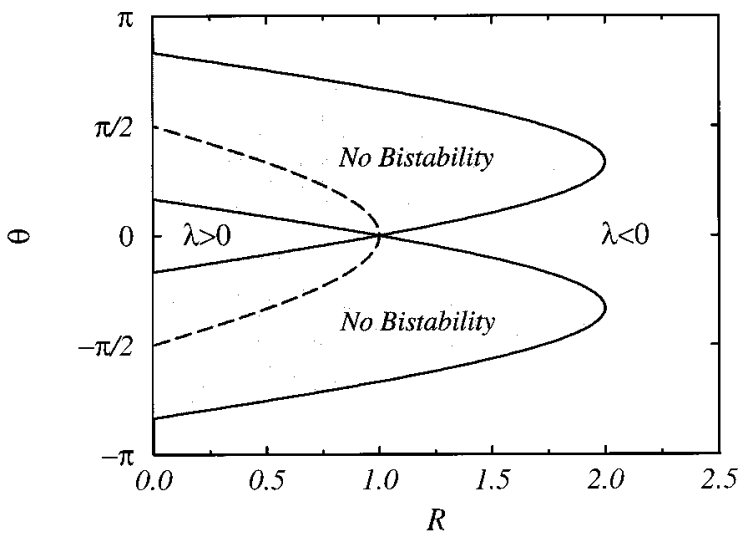

FIG. 4. The phase diagram for the onset of bistability for a $\delta$ function sandwiched between two linear systems, as derived from Eqs. (13) and (14). $\theta$ and $R$ are defined through $1 / T_{0}=R e^{i \theta} / T$ where $T_{0}$ is the total linear transmission amplitude of the two linear structures with the $\delta$ function in the middle $(\lambda \equiv 0)$ and $T$ is the total transmission amplitude without the $\delta$ function $\left(\epsilon_{0} \equiv 0\right)$. Eq. (13) sets the values of $R$ and $\theta$ for which we can observe bistability, outside the gray areas, while Eq. (14) (dashed line) sets the sign of the nonlinear coefficient $\lambda$ for which we will observe bistability.

$$
\begin{aligned}
& \frac{\gamma}{\tau^{\prime}}<\frac{1}{3}, \\
& \lambda^{\prime}<0,
\end{aligned}
$$

thus, allowing bistability for positive Kerr coefficients as well. We want to stress that the conditions for bistability depend on the transmission amplitudes of the linear systems with and without $\delta$ function only. They can conveniently be rewritten in terms of the quantities $R$ and $\theta$ which are defined via $1 / T_{0}=R e^{i \theta} / T$ :

$$
\begin{gathered}
\frac{\sin ^{2} \theta}{(R-\cos \theta)^{2}} \leqslant \frac{1}{3}, \\
\operatorname{sign}(\lambda)\left(1-\frac{\cos \theta}{R}\right)<0 .
\end{gathered}
$$

Figure 4 shows the phase diagram for the onset of bistability derived from Eqs. (13) and (14). Obviously, the insertion of the linear part of the nonlinear $\delta$ function has to sufficiently alter the total transmission amplitude $1 / T$ in order for bistability to occur. This alteration may be achieved by a change in the total transmission, i.e., by changing $R$, and/or by introducing a phase shift $\theta$.

One can get more explicit and transparent results when the linear systems each consist of a periodic arrangement of $N$ linear $\delta$ functions with a spacing $a$ equal to the distance between the nonlinear $\delta$ function and the two linear systems. We first consider the linear problem of $N$ equally spaced $\delta$ functions of dielectric strength $\epsilon_{0}$. Between any two $\delta$ functions in the linear structure the solution consists of two plane waves traveling in opposite directions. Using the boundary conditions we can eliminate the waves in the intermediate space and obtain a difference equation in terms of the field at the $\delta$-function sites, ${ }^{17}$

$$
E_{n+1}+E_{n-1}=\left(2 \cos k-\epsilon_{0} k \sin k\right) E_{n},
$$

where we have assumed the space between two $\delta$ functions to be vacuum and their separation to be $a=1$. Upon expressing all lengths in units of the lattice period $(a=1)$ and using the Bloch's theorem $E(x+1)=e^{i q} E(x)$ we arrive at the band-structure equation that defines the Bloch wave vector $q$,

$$
\cos q=\cos k-\frac{1}{2} \epsilon_{0} k \sin k .
$$

The transmission bands are found by setting $|\cos q| \leqslant 1$.

We can easily generalize the above method to the present problem of $2 N+1 \delta$ functions utilizing the transfer matrix of each layer,

$$
\left(\begin{array}{c}
E_{-N-1} \\
E_{-N}
\end{array}\right)=\mathcal{M}^{N}\left(\begin{array}{cc}
2 \cos q^{\prime} & -1 \\
1 & 0
\end{array}\right) \mathcal{M}^{N}\left(\begin{array}{c}
E_{N} \\
E_{N+1}
\end{array}\right),
$$

where

$$
\mathcal{M}=\left(\begin{array}{cc}
2 \cos q & -1 \\
1 & 0
\end{array}\right)
$$

and $2 \cos q^{\prime}=2 \cos k-\epsilon_{0}^{\prime}\left(1+\lambda|E(0)|^{2}\right) k \sin k$, comes from the nonlinear $\delta$ function at $x=0 . E_{-N}$ and $E_{-(N+1)}$ are given by the output field,

$$
E_{-N}=E_{t} e^{-i N k}, \quad E_{-(N+1)}=E_{t} e^{-i(N+1) k} .
$$

$E_{N}$ and $E_{N+1}$ are related to the incoming and reflected fields by

$$
\begin{gathered}
E_{N}=E_{0} e^{-i N k}+E_{r} e^{i N k}, \\
E_{N+1}=E_{0} e^{-i(N+1) k}+E_{r} e^{i(N+1) k} .
\end{gathered}
$$

The intensity at the nonlinear site $|E(0)|^{2}$ can be expressed in terms of the transmitted intensity $\left|E_{-N}\right|^{2}=X$ as $|E(0)|^{2}=C X$, where

$$
C=1 \mp \epsilon_{0} k \sin k \frac{\sin N q \sin (N+1) q}{\sin ^{2} q} .
$$

In Eq. (22) $q$ should be replaced by $i|q|$ for $|\cos q|>1$, and the lower sign in $C$ is used when $\cos q<-1$. After some algebra we arrive at the final result,

$$
Y=X\left[1+\tau^{\prime}\left(1+\lambda^{\prime} X\right)^{2}\right]
$$

where

$$
\tau^{\prime}=\tau \alpha^{2} \frac{\sin ^{2}(2 N+1) q}{\sin ^{2} q}, \quad \lambda^{\prime}=\lambda \frac{\epsilon_{0}^{\prime}}{\epsilon_{0}} \alpha^{-1} \frac{C^{2} \sin q}{\sin (2 N+1) q},
$$

and

$$
\alpha=\left(1+\frac{\left(\epsilon_{0}^{\prime}-\epsilon_{0}\right) C \sin q}{\epsilon_{0} \sin (2 N+1) q}\right) .
$$

Of course, this result can also be obtained from Eqs. (6)-(8) by specifying the respective expressions for the reflection and transmission amplitudes, $r_{i}$ and $t_{i}(i=1,2)$. Since Eq. (2) and Eq. (23) have the same form, all the analysis done in Sec. II A applies. However, due to the renormalization of the parameters $\tau$ and $\lambda$, interesting features arise: $\tau^{\prime}$ is related to 


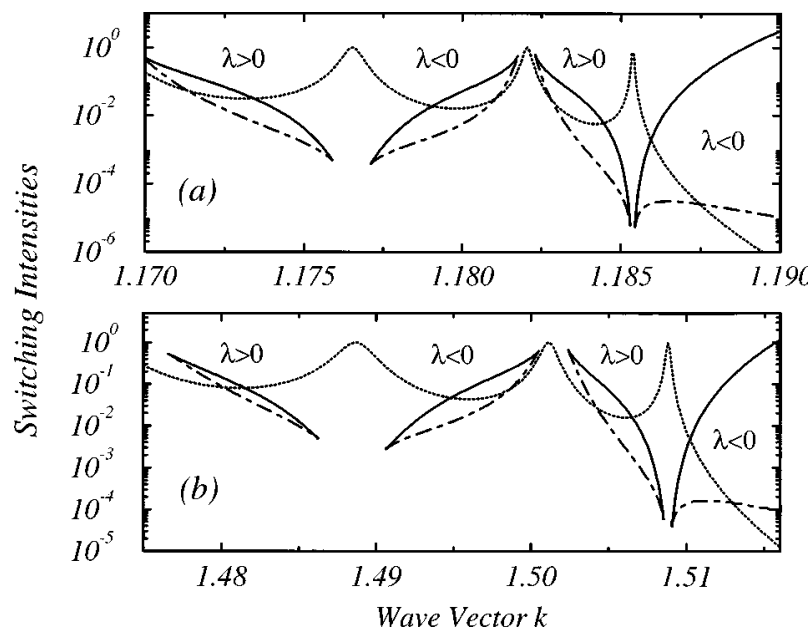

FIG. 5. Switching-up (solid line) and switching-down (dotdashed line) intensities for a nonlinear impurity system sandwiched between two linear structures. The linear structures consist of (a) $\delta$ functions with $N=20, \epsilon_{0}^{\prime}=\epsilon_{0}=2.5$, and spacing $a=1$, and as a comparison, (b) a system of 41 dielectric bilayers with $d_{a}=d_{b}=0.5$ and $n_{a}^{2}=1, n_{b}^{2}=5$ in analogy with the $\delta$-function system: $d_{b} n_{b}^{2}=\epsilon_{0}$. The $b$ layer in the middle of the structure is nonlinear with $\epsilon_{b}(x)=n_{b}^{2}\left(1+\lambda|E(x)|^{2}\right)$. In both cases we used $\lambda= \pm 1$. The dotted line in both graphs is the linear transmission coefficient of the structure. The band gap starts around $k=1.186$ for the $\delta$-function model and $k=1.51$ for the finite-width model, as can be seen from the large transmission drop. The exact value of the band edge can be found by using Eq. (16)

the total linear $(\lambda=0)$ transmission coefficient of the system $\tau^{\prime}=\left(1-\left|T_{\text {TOTAL }}\right|^{2}\right) /\left|T_{\text {TOTAL }}\right|^{2}$, and the condition $\tau^{\prime}>3 \mathrm{im}-$ plies $\left|T_{\text {TOTAL }}\right|^{2}<0.25$. Also, the constraint $\lambda<0$ is now relaxed since it requires only $\lambda^{\prime}<0$.

In Fig. 5(a) we plot the switching up and down intensities for the case $\epsilon_{0}^{\prime}=\epsilon_{0}$ and for frequencies close to the first band gap. We note that the sign of $\lambda$ required to obtain bistability, between successive lobes of the linear $(\lambda=0)$ transmission curve, is alternating from $\lambda<0$ to $\lambda>0$. We can understand this by means of the field configuration that characterizes these lobes for the linear lattice. Every lobe corresponds to a transmission resonance for which the intensity envelope is $|E(x)|^{2} \propto \sin ^{2}(m x / L)$ where $L$ is the length of the structure, $0 \leqslant x \leqslant L$, and $m=1,2,3, \ldots 2 N+1$ starting from the upper band edge and moving to lower frequencies. ${ }^{19}$ When $m$ is odd, the intensity at the middle of the structure will have a maximum, while when $m$ is even, it will have a minimum. If we now turn on the nonlinearity $(\lambda \neq 0)$ in the $\delta$ function in the middle of the structure, it will introduce an appreciable phase shift only when the incident wave is tuned close to an "odd" lobe's frequency. This phase shift will change the intensity envelope, and thus the resonance frequencies, resulting in an effective frequency "shift" for the "odd", lobes. For some intensity, the incident wave's frequency will match to the "shifted" lobe's frequency, and a transmission resonance will be obtained. This is where the bistable behavior originates from. The condition on $\tau^{\prime}$ sets the allowed frequencies for observing bistability in between the transmission lobes. Also, a negative nonlinearity $(\lambda<0)$ will cause the odd lobes to shift to higher frequencies, while a positive nonlinearity $(\lambda>0)$ will cause them to shift to lower
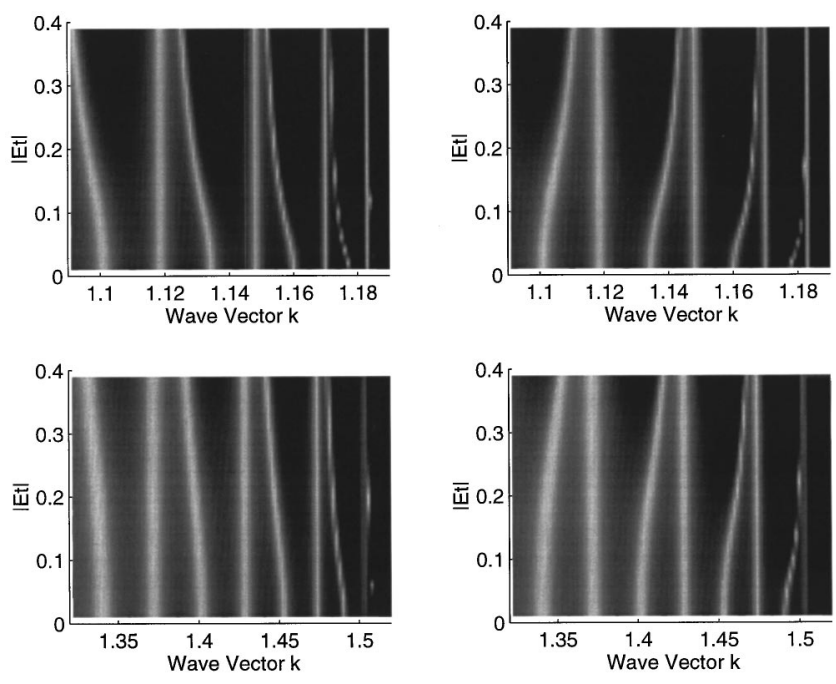

FIG. 6. Gray scale plots of the transmission coefficient vs the wave vector $k$ and the transmitted amplitude $\left|E_{t}\right|$ for frequencies inside the transmission band. Dark areas indicate low transmission. The two upper graphs correspond to the $\delta$-function model described in Fig. 5(a), for $\lambda=1$ (left) and $\lambda=-1$ (right). The two lower graphs correspond to the finite-width model described in Fig. 5(b), for $\lambda=1$ (left) and $\lambda=-1$ (right). The transmission lobes and their frequency shift are clearly shown, except for the first one right on the band edge, which can barely be distinguished for the $\lambda=1$ case, while for $\lambda=-1$ it shifts inside the band gap.

frequencies. ${ }^{19}$ This is shown in Fig. 6 for both the $\delta$ function and finite-width models. Note that lobes closer to the gap shift at lower intensities, indicating that for those frequencies smaller switching thresholds should be expected. With these features, the bistability diagram in Fig. 5(a) is fully understood.

An important consequence of the introduction of the two linear systems is the presence of resonances. The phase shift introduced by the nonlinear $\delta$ function must be enough to tune the incident wave with the shifted lobe. The same transmission lobes are found in the linear transmission diagram of a finite layer superlattice. Then, from the point of view of induced phase shifts, there should be no real qualitative difference between the $\delta$-function model and the finite layer superlattice, at least for the first bistable loop. The finite layer system differs qualitatively from the nonlinear $\delta$-function model insofar as it always exhibits multistability (the nonlinear $\delta$-function system is strictly bistable), a point that will be discussed in Sec. III. But when considering the first bistable loop only, the very thin layer approximation may be viewed as a reliable guide to more realistic systems involving finite-width nonlinear layers. This is shown in Fig. 5(b) where we plot the switching up and down intensities of the first bistable loop, for a finite-width layered model with a finite-width nonlinear impurity layer, having its system parameters defined in correspondence with the $\delta$-function model by $\epsilon_{0}=d n^{2}$. In order to stress the similarity, we have chosen the nonlinear layer to equal to half a lattice period. We see that the $\delta$-function model indeed captures most of the essential features of the more realistic finite width superlattice.

Similar behavior is obtained for the $\epsilon_{0}^{\prime} \neq \epsilon_{0}$ impurity case for frequencies inside the transmission band. In Fig. 7 we 


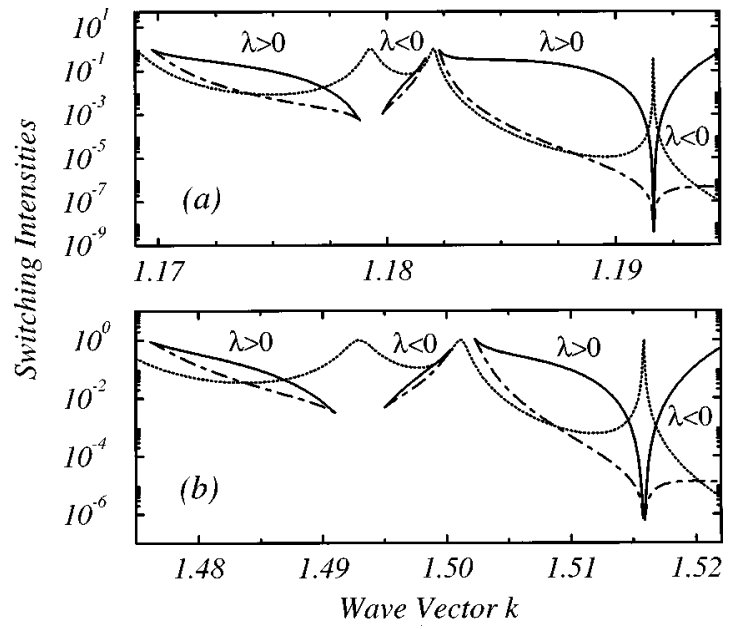

FIG. 7. The switching intensities for the same systems as described in Fig. 5, but now the nonlinear impurity is (a) a nonlinear $\delta$ function with $\epsilon_{0}^{\prime}=2.2$ and (b) a nonlinear layer $\epsilon(x)=n_{d}^{2}\left(1+\lambda|E(x)|^{2}\right)$ with $n_{d}^{2}=4.4$. We again used $\lambda= \pm 1$. The first lobe, that shifted in the gap and is now the linear $(\lambda=0)$ impurity mode, is clearly seen in both cases. Note how the switching intensities dropped about three orders of magnitude around this mode.

show the switching intensities of both the $\delta$-function layer and finite-width layer systems for a weak impurity $\epsilon_{0}^{\prime}<\epsilon_{0}$. For these values, the linear $(\lambda=0)$ impurity mode, which is actually the $m=1$ transmission lobe being shifted inside the band gap, ${ }^{19}$ is very close to the transmission band. For $\epsilon_{0}^{\prime}>\epsilon_{0}$, no gap impurity mode exists in the $\delta$-function model so the response of the two systems inside the gap is different. For $\epsilon_{0}^{\prime}<\epsilon_{0}$, both systems exhibit an impurity mode inside every band gap. ${ }^{14}$ When the fields are turned on, a positive nonlinearity in the middle of the structure will shift the impurity mode to lower frequencies, while a negative nonlinearity will shift it to higher frequencies. As a consequence, when $\lambda>0$ bistability is observed at frequencies lower than that of the linear impurity mode, while for $\lambda<0$ we must use higher frequencies.

The switching up and down intensities are generally $Y_{-} \sim 1 /\left|\lambda^{\prime}\right|$ and $Y_{+} \sim \tau^{\prime} /\left|\lambda^{\prime}\right|$, and for band-gap frequencies it is generally $\tau^{\prime} \gg \lambda^{\prime} \gg 1$, yielding extremely low switchingdown intensities and very high switching-up intensities. Nevertheless, for frequencies relatively close to the linear defect frequency, it is $\lambda^{\prime} \gg \tau^{\prime} \gg 1$, yielding extremely small switching up intensities as well. As can be seen in Fig. 8, where we plot the switching intensities for a large impurity (linear impurity mode deep inside the band gap), the order of magnitude for a $N=20$ system is well below $10^{-20}$, and becomes exponentially small as $N$ gets larger. To get a feeling for this number, assume that typical electronic nonlinearities are of the order of $|\lambda| \sim 10^{-15} \mathrm{~cm}^{2} / \mathrm{W}$. Then $Y_{ \pm} \sim 10^{-5} \mathrm{~W} / \mathrm{cm}^{2}$. Furthermore, the intensity at the nonlinear defect layer is small enough, to secure that the nonlinearity is well described as a Kerr nonlinearity: The nonlinear effect does not saturate and the nonlinear layer will not get damaged from intense fields. However, the price one has to pay to achieve low power thresholds, is to maintain extreme accuracy in the incident frequency, due to the extremely high $Q$ of the mode. For the system described in Fig. 8 this accuracy is of the

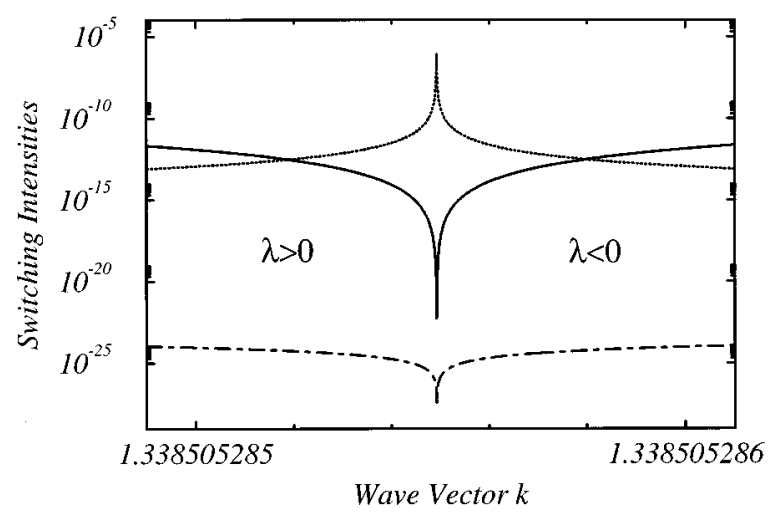

FIG. 8. The switching intensities (solid and dot-dashed lines for switching up and down, respectively) for the same system of Fig. 5 (a) for the $\delta$-function model but now for the large impurity case $\epsilon_{0}^{\prime}=1$. The linear impurity mode is now deep in the gap (dotted line corresponds to the linear transmission coefficient). Note that for frequencies very close to this mode the switching intensities become extremely small.

order of $\Delta k / k \sim 10^{-10}$. This is quite unrealistic. In addition, this ratio gets exponentially small for increasing $N$. Thus, for a realistic application it is the laser's linewidth that determines the power thresholds for bistability and in general compromise has to be found.

\section{DIELECTRIC SUPERLATTICE WITH NONLINEAR IMPURITY: RESONANCE STATES}

In the previous section, we have investigated the response of a very thin layer of nonlinear material sandwiched between two linear Bragg-reflector structures. We demonstrated that such a structure can exhibit bistability with very low switching threshold as a result of coupling to the impurity mode in the stop gap of the linear structure. In this section, we consider the situation when the nonlinear layer is not thin. There are qualitative differences between the response of a nonlinear finite-width layer and a nonlinear $\delta$-function layer. We have seen that a single finite-width dielectric layer exhibits bistability for both positive and negative Kerr coefficients [Fig. 2(a)], whereas the single nonlinear $\delta$ function exhibits bistability for negative Kerr coefficient only. Similarly, and in contrast to the strictly bistable $\delta$-function model, dielectric superlattices with a nonlinear impurity always exhibit multistability. This, we will show, has to do with the fact that impurity modes exist for each value of $\Delta \epsilon$. We can understand all these properties qualitatively if we view the finite nonlinear layer as consisting of a sequence of of nonlinear $\delta$ functions. Then, resonance phenomena allow to bypass the limited behavior of a single nonlinear $\delta$ function. However, multistability is much harder to detect than bistability, because the field values at the nonlinear layer may be very large and oscillating, thus requiring extremely high precision.

Let us first investigate the localized mode solution for a finite-width nonlinear layer sandwiched between two Bragg reflectors. The nonlinear wave equation inside the impurity layer is given by 


$$
\frac{d}{d x^{2}} E(x)+k_{0}^{2}\left[1+\lambda|E(x)|^{2}\right] E(x)=0,
$$

where $k_{0}=n^{2}(\omega / c)$. This equation may be solved by means of the following ansatz:

$$
E(x)=E_{0} g(x) e^{i \phi(x)} .
$$

Inserting this ansatz into Eq. (24) leads to a separation of the amplitude and phase function, $g(x)$ and $\phi(x)$, respectively:

$$
\begin{gathered}
\frac{d}{d x} \phi(x)=\frac{W}{g^{2}(x)}, \\
\left(\frac{d g(x)}{d x}\right)^{2}+\frac{W}{g^{2}(x)}+k_{0}^{2} g^{2}(x)+\frac{1}{2} \widetilde{\lambda k_{0}^{2} g^{4}(x)=A,}
\end{gathered}
$$

where $\tilde{\lambda}=\lambda\left|E_{0}\right|^{2}$ is the effective nonlinearity and $A$ and $W$ are constants to be determined. Upon introducing $I(x)=g^{2}(x)$, the solution may be cast in the deceptively simple form

$$
\begin{gathered}
\int_{I\left(x_{0}\right)}^{I(x)} \frac{d I}{\left(A I-k^{2} I^{2}-\frac{1}{2} \widetilde{\left.\lambda k^{2} I^{3}-W^{2}\right)^{1 / 2}}= \pm 2\left(x-x_{0}\right),\right.} \\
\phi(x)=\phi\left(x_{0}\right)+W \int_{x_{0}}^{x} d x^{\prime} \frac{1}{I\left(x^{\prime}\right)} .
\end{gathered}
$$

The four unknowns $A, W, \phi\left(x_{0}\right), I\left(x_{0}\right) \equiv g^{2}\left(x_{0}\right)$ have to be determined from the boundary conditions at $x_{0}$. In particular, $W$ is related to the energy flux through the layer as can be seen by evaluating the Poynting "vector", $S=-c^{2} / 8 \pi \omega \operatorname{Re}\left[i E^{*}(x)(d E(x) / d x)\right]=c^{2}\left|E_{0}\right|^{2} W / 8 \pi c$. In the case of $\lambda=0$ it is an easy exercise to obtain the linear solutions from Eq. (28). For $\lambda \neq 0$, despite the apparent simplicity of Eq. (28), a closed form solution cannot be obtained in general. The reason for this difficulty is seen as follows: The solution of Eq. (28) comes down to finding the roots of the denominator, where $A$ and $W$ depend on the boundary values in a complicated way. This task can be accomplished in two special circumstances only: If $d \phi\left(x_{0}\right) / d x=0$, then, according to Eq. (27) we already have one root, leaving us with the simple exercise of solving a quadratic equation. Similarly, if $W=0$ we immediately have the root $I=0$, again reducing the problem to a quadratic equation. The first case was exploited by Chen and Mills ${ }^{6}$ in solving the transmission problem through a single finite-width nonlinear layer only, without the superlattices on both sides.

We, however, are interested in the second situation. Apparently, $W=0$ implies $S=0$. This corresponds to a spatially symmetric situation, i.e., a situation where parity is a good "quantum', number. Speaking in terms of the transmission experiment we have in mind, this amounts for solving for the stationary or resonance states for which input equals output. This distinction is of no importance in the linear problem, because there we can construct any state by an appropriate superposition of stationary states. Obviously, this cannot be done in a nonlinear problem. Consider now the case where the nonlinear layer, centered at $x=0$, is sandwiched between two infinitely extended linear superlattices of alternating lay-

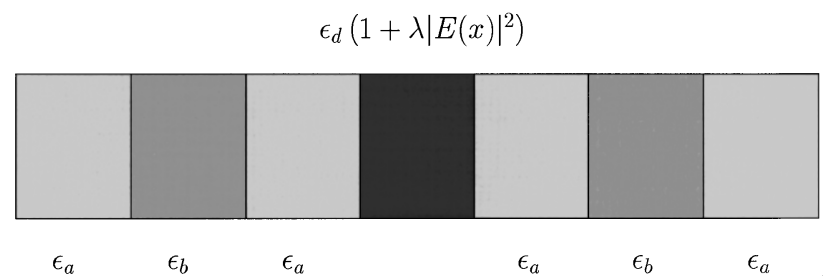

FIG. 9. The model superlattice used in this section. A nonlinear layer of dielectric constant $\epsilon_{d}\left(1+\lambda|E(x)|^{2}\right)$ and thickness $\ell$ centered at $x=0$ is sandwiched between two infinitely extended superlattices of alternating layers with dielectric constants $\epsilon_{a}$ and $\epsilon_{b}$, and widths $a$ and $a-d$, respectively.

ers of dielectric constants $\epsilon_{a}$ and $\epsilon_{b}$ and widths $a-d$ and $d$, respectively (cf. Fig. 9). Since the stationary states have parity $p= \pm 1$ we may impose the following values of the electric field $E(0)$ and its derivative $E^{\prime}(0)$, i.e., the magnetic field, at the origin.

Even solution $(p=1)$ :

$$
\begin{gathered}
E(0)=E_{0} \neq 0 \Rightarrow \phi(0)=0, \quad g(0)=1 \\
E^{\prime}(0)=0 \Rightarrow g^{\prime}(0)=0 .
\end{gathered}
$$

Odd solution $(p=-1)$ :

$$
\begin{gathered}
E(0)=0 \Rightarrow \phi(0)=0, \quad g(0)=0 \\
E^{\prime}(0)=E_{0} k_{0} \sqrt{1+\tilde{\lambda} / 2} \neq 0 \Rightarrow g^{\prime}(0)=k_{0} \sqrt{1+\tilde{\lambda} / 2} .
\end{gathered}
$$

Here, we have chosen the nonzero value of the field's derivative for the odd solution in a particular convenient form (compare with the later calculations for the constant $A$ ).

To compare with numerical studies, the above equations define the value of $E_{0}$ and, thus, the effective nonlinearity $\tilde{\lambda}$. Consequently, for a given frequency we need to search for the symmetric states (resonant states) in the transmission problem and compute the field $E_{0}$ at the origin. For given $\lambda$ we then get the effective nonlinearity $\widetilde{\lambda}=\lambda\left|E_{0}\right|^{2}$.

The computation of the parameter $A$ in Eq. (27) is now straightforward and due to the "tricky" definitions is the same for both parities:

$$
A=k_{0}^{2}\left(1+\frac{1}{2} \tilde{\lambda}\right)
$$

In addition, we can compute the missing roots of the cubic expression in Eq. (28):

$$
\begin{gathered}
I_{1}=0, \\
I_{2}=-\frac{2+\tilde{\lambda}}{\tilde{\lambda}}, \\
I_{3}=1 .
\end{gathered}
$$

In order to finally solve Eq. (28), we have to distinguish four cases: The solution depends on parity, and the sign of one root, namely, $I_{2}$ depends on the sign of $\tilde{\lambda}$ (cf. Ref. 20). We begin with $\tilde{\lambda>0}$ : Define the quantities 


$$
\begin{gathered}
\kappa=\sqrt{\frac{\tilde{\lambda}}{2+2 \tilde{\lambda}}}, \\
\alpha=\left\{\begin{array}{cc}
K(\kappa), \quad p=1 \\
0, \quad p=-1,
\end{array}\right.
\end{gathered}
$$

where $K$ denotes the complete elliptic integral of the first kind. Then, the solution to Eq. (28) reads as

$$
I(x)=\frac{\operatorname{sn}^{2}\left( \pm k_{0} x \sqrt{1+\tilde{\lambda}}+\alpha ; \kappa\right)}{1+\frac{\tilde{\lambda}}{2+\tilde{\lambda}} \operatorname{cn}^{2}\left( \pm k_{0} x \sqrt{1+\tilde{\lambda}}+\alpha ; \kappa\right)} .
$$

Here, sn and cn are the Jacobian elliptic functions. These results may be simplified by the use of the addition theorems of the Jacobian elliptic functions.

$$
\begin{gathered}
p=1: \\
\quad I(x)=\operatorname{cn}^{2}\left(k_{0} x \sqrt{1+\tilde{\lambda} ; \kappa) .}\right. \\
I(x)=\left(\frac{1+\frac{1}{2} \tilde{\lambda}}{1+\tilde{\lambda}}\right) \frac{\operatorname{sn}^{2}\left(k_{0} x \sqrt{1+\tilde{\lambda}} ; \kappa\right)}{\operatorname{dn}^{2}\left(k_{0} x \sqrt{1+\tilde{\lambda} ; \kappa}\right)},
\end{gathered}
$$

where $\mathrm{dn}=\sqrt{1-\kappa^{2} \mathrm{sn}^{2}}$. Similar solutions emerge in the case of a negative Kerr coefficient, $\tilde{\lambda}<0$. Define the quantities

$$
\begin{gathered}
\eta=\mid \tilde{\lambda \mid}, \\
\kappa=\sqrt{\frac{\eta}{2-\eta}} \\
\beta=\left\{\begin{array}{cc}
K(\kappa), & p=1 \\
0, & p=-1 .
\end{array}\right.
\end{gathered}
$$

Then the solution to Eq. (28) reads as

$$
I(x)=\operatorname{sn}^{2}\left( \pm k_{0} x \sqrt{1-\frac{1}{2} \eta}+\beta ; \kappa\right) .
$$

These results may, again, be simplified by the use of the addition theorems of the Jacobian elliptic functions

$$
\begin{aligned}
& p=1: \\
& \quad I(x)=\frac{\operatorname{cn}^{2}\left(k_{0} x \sqrt{1-\frac{1}{2} \eta} ; \kappa\right)}{\operatorname{dn}^{2}\left(k_{0} x \sqrt{1-\frac{1}{2} \eta} ; \kappa\right)} . \\
& p=-1: \quad I(x)=\operatorname{sn}^{2}\left(k_{0} x \sqrt{1-\frac{1}{2} \eta} ; \kappa\right) .
\end{aligned}
$$

Outside the nonlinear layer the waves obey the BlochFloquet theorem. We employ the "traveling wave", description, ${ }^{21}$ which decomposes the field $E(x)$ and (implicitly) its derivative $E^{\prime}(x)$ into left- and right-moving waves:

$$
\vec{E}(x)=\left(\begin{array}{c}
A_{r} e^{i k x} \\
A_{l} e^{-i k x}
\end{array}\right) .
$$

Within this formalism, the Bloch-Floquet condition for a defect state inside the photonic band gap, created by the linear superlattice, may be written as (connecting the fields in the middle of one linear $A$ layer at $x=s$ to the fields at $x=a+s$, where $a$ is a lattice constant):

$$
\left(\mathcal{M} \pm e^{-\gamma a} \mathcal{E}\right) \vec{E}(s)=0
$$

where the matrix elements of $\mathcal{M}$ are well known:

$$
\begin{gathered}
\mathcal{M}_{11}=e^{i k_{a}(a-d)}\left(\cos \left(k_{b} d\right)+\frac{i}{2}\left(\frac{k_{b}}{k_{a}}+\frac{k_{a}}{k_{b}}\right) \sin \left(k_{b} d\right)\right), \\
\mathcal{M}_{12}=\frac{i}{2}\left(\frac{k_{b}}{k_{a}}-\frac{k_{a}}{k_{b}}\right) \sin \left(k_{b} d\right) .
\end{gathered}
$$

where $k_{a}=(\omega / c) \sqrt{\epsilon_{a}}, \quad k_{b}=(\omega / c) \sqrt{\epsilon_{b}}$ and $\mathcal{M}_{11}=\mathcal{M}_{22}^{*}$, $\mathcal{M}_{12}=\mathcal{M}_{21}^{*}$. Inside the nonlinear layer we know the field and its derivative at the origin. Using the solutions Eqs. (33)-(39), we can now calculate the field and its derivative at the interface to the $A$ material, translate the results into the traveling wave formalism (field and derivative are continuous at the boundary!) and propagate them to the middle of the $A$ layer, i.e., we obtain $\vec{E}(s)$ :

$$
\begin{gathered}
(\vec{E})_{1}(s)=e^{i k_{a} s} \sqrt{I(l / 2)}-\frac{i}{k_{a}} e^{i k_{a} s} \frac{d}{d x} \sqrt{I(l / 2)}, \\
(\vec{E})_{2}(s)=\left[(\vec{E})_{1}(s)\right]^{*},
\end{gathered}
$$

where $s=(a-l) / 2$ and $l$ is the thickness of the nonlinear layer. Thus, Eq. (41) constitutes two linear equations, the second being the complex conjugate of the first. Upon separating this complex equation into real and imaginary parts, we observe that the real part does not contain $\gamma$ and may thus be used to determine $\omega$ :

$$
\begin{aligned}
& \sin \left[k_{a}(a-d)\right] \cos \left(k_{b} d\right)+\frac{1}{2}\left(\frac{k_{b}}{k_{a}}+\frac{k_{a}}{k_{b}}\right) \cos \left[k_{a}(a-d)\right] \\
& \times \sin \left(k_{b} d\right)+\frac{1}{2}\left(\frac{k_{b}}{k_{a}}-\frac{k_{a}}{k_{b}}\right) \sin \left(k_{b} d\right) \psi(\omega)=0 .
\end{aligned}
$$

Here, $\psi(\omega)$ contains the information about the nonlinear layer. If we define

$$
\begin{gathered}
\chi^{(0)}(x)=\frac{\sqrt{I(x)}}{k_{a}} \frac{d(\sqrt{I(x)})}{d x}, \\
\chi^{(+/-)}(x)=I(x) \pm \frac{1}{k_{a}^{2}}\left(\frac{d(\sqrt{I(x)})}{d x}\right)^{2},
\end{gathered}
$$

then $\psi(\omega)$ is given as

$$
\psi(\omega)=\frac{\cos \left(k_{a} s\right) \chi^{(-)}(l / 2)+2 \sin \left(k_{a} s\right) \chi^{(0)}(l / 2)}{\chi^{(+)}(l / 2)},
$$

where $s=a-d+l$ ( $a=$ lattice constant; $d=$ thickness of the $B$ layers; $l=$ thickness of the nonlinear layer). $\sqrt{I(l / 2)}$ and $d(\sqrt{I(l / 2)}) / d x$ have to be evaluated according to the sign of $\tilde{\lambda}$ and parity. 


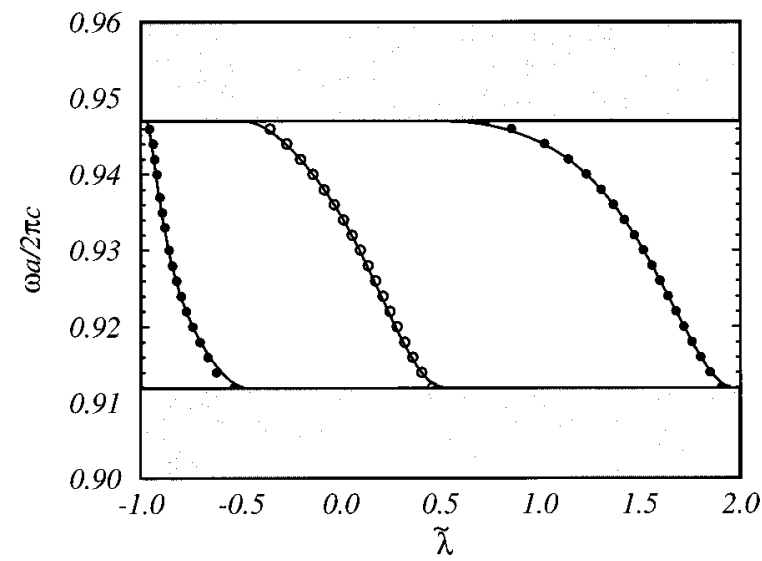

FIG. 10. The resonance state frequencies as a function of the effective nonlinearity $\tilde{\lambda}$ inside the third band gap. The linear superlattice consists of equal thickness layers of alternating dielectric constants $\epsilon_{a}=1$ and $\epsilon_{b}=5$, while the nonlinear layer has $\epsilon_{d}=8$, $\ell=d$, and $\lambda=1$. Solid lines correspond to the analytical results while circles correspond to the results of a numerical simulation using 20 bilayers on each side of the nonlinear layer. Filled circles correspond to states with even parity, while open circles correspond to states with odd parity. The gray areas correspond to parts of the third and fourth transmission bands.

Equation (44) defines the solution for the impurity modes for our structure. These impurity modes manifest themselves as resonances in the nonlinear multistable response, for frequencies inside the gap of the linear superlattice. In the $\delta$-function model we had only one such resonance, as required from its strictly bistable character. As discussed above, for a nonlinear impurity of finite width in an infinitely extended linear superlattice we now expect multistable behavior. For practical applications, the linear superlattices cannot be infinitely extended. However, as long as the number of the layers is large enough to have a well-defined localized solution, the resonant frequencies should be given exactly by Eq. (44).

Figure 10 shows the resonance state frequencies as a function of $\tilde{\lambda}$ for a linear superlattice consisting of equally wide layers of alternating dielectric constant $\epsilon_{a}=1$ and $\epsilon_{b}=5$. The nonlinear layer is described by $n^{2}=8, l=d$, and $\lambda=1$. Solid lines represent the analytical solution according to Eq. (44), while the circles correspond to the results of a numerical simulation using 20 bilayers on each side of the nonlinear layer. In addition, the parity of the resonance states is indicated. Evidently, the agreement between the two methods is excellent, thus illustrating the multistable behavior. This feature (multistability) is explicitly shown in Fig. 11, where we show the input-output diagram for the same configuration. Finally, in Fig. 12 we show the intensity configuration for two resonance states with different parities. Each resonance state is characterized by the intensity configuration inside the nonlinear layer. The first has one lobe, the second two, the third three, etc. In the $\delta$-function case, the possibility of different intensity configurations inside the nonlinear medium is absent. Thus, only one resonance state would be allowed, which is the analogue of the first resonance state of the finite-width case shown in Fig. 12(a).

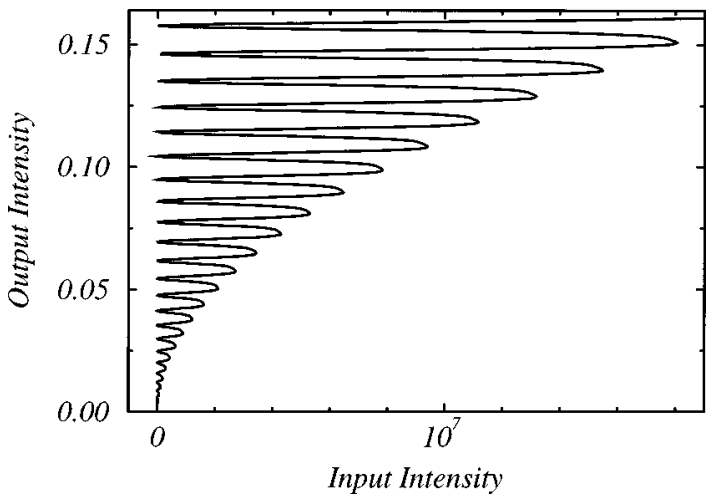

FIG. 11. Transmitted intensity vs incident intensity for the same system described in Fig. 10. We used a model of 20 bilayers on each side of the nonlinear layer with $\lambda=1$ and frequency $\omega a / 2 \pi c=0.93$. Every resonance point in this diagram (output =input) will yield a point in the resonance frequency diagram in Fig. 10, with alternating parity. The very first resonance (not distinguishable in this picture) corresponds to an open circle in Fig. 10 for $\omega a / 2 \pi c=0.93$ and will have an odd parity.

\section{CONCLUSIONS}

We have investigated the general problem of electromagnetic wave propagation through a one-dimensional system consisting of a nonlinear layer sandwiched between any two linear systems. Our studies provide a general frame within which calculations can be done, based on the transmission characteristics of the linear systems. In the case of a very thin layer, we have shown that a $\delta$-function approximation is adequate. It follows from our analysis that a nonlinear layer sandwiched between two identical Bragg reflectors can be

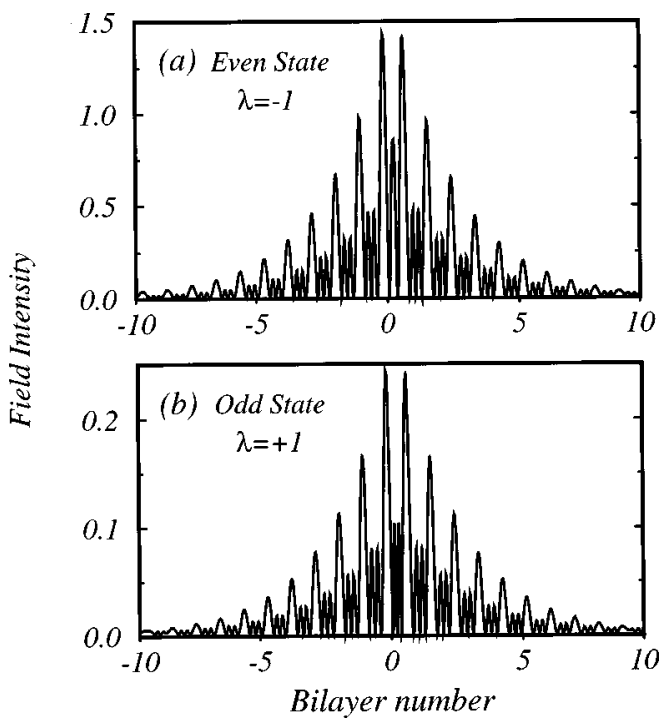

FIG. 12. We plot the intensity configuration for the same model described in Fig. 10, for $\omega a / 2 \pi c=0.93$. (a) Even-parity state with $\lambda=-1$, corresponding to the branch on the left (filled circles) in Fig. 10. (b) Odd-parity state with $\lambda=1$, corresponding to the branch in the middle of Fig. 10. The latter is actually the very first resonance state described in Fig. 11. 
regarded as the most efficient "localized" feedback structure. For this system, a cubic equation has been derived, describing the nonlinear bistable response, with normalized parameters that characterize the experimental situation. We obtain bistability in the gap of the linear system, via the impurity mode, and find that the switching thresholds can be made very small by enlarging the structure and/or widening the gap. In addition, we have obtained an analytic solution for the localized resonance modes in the band gap for a nonlinear impurity of finite extent in an otherwise linear dielectric superlattice. The solution shows multistable behavior, the basic feature absent in the $\delta$-function model. Our numerical studies agree extremely well with these solutions.

\section{ACKNOWLEDGMENTS}

Ames Laboratory is operated for the U.S. Department of Energy by Iowa State University under Contract No. W7405-Eng-82. This work was supported by the Director for Energy Research, Office of Basic Energy Sciences and Advanced Energy Projects and by NATO Grant No. 940647. K.B. acknowledges financial support from the GermanIsraeli Foundation (GIF).
${ }^{1}$ H. G. Winfull, J. H. Marburger, and E. Garmire, Appl. Phys. Lett. 35, 379 (1979).

${ }^{2}$ W. Chen and D. L. Mills, Phys. Rev. B 35, 524 (1987).

${ }^{3}$ H. M. Gibbs, S. L. McCall, and T. N. C. Venkatesan, Phys. Rev. Lett. 36, 1135 (1976).

${ }^{4}$ B. Xu and N. B. Ming, Phys. Rev. Lett. 71, 3959 (1993).

${ }^{5}$ C. J. Herbert, W. S. Capinski, and M. S. Malcuit, Opt. Lett. 17, 1037 (1992).

${ }^{6}$ W. Chen and D. L. Mills, Phys. Rev. B 36, 6269 (1987).

${ }^{7}$ F. S. Felber and J. H. Marburger, Appl. Phys. Lett. 28, 731 (1976).

${ }^{8}$ M. Okuda and K. Onaka, Jpn. J. Appl. Phys. 16, 769 (1977).

${ }^{9}$ F. Deylon, Y.-E. Levy, and B. Souillard, Phys. Rev. Lett. 57, 2010 (1986).

${ }^{10}$ Yi Wan and C. M. Soukoulis, Phys. Rev. A 41, 800 (1990).

${ }^{11}$ D. Henning, H. Gabriel, G. P. Tsironis, and M. Molina, Appl. Phys. Lett. 64, 2934 (1994).

${ }^{12}$ M. I. Molina and G. P. Tsironis, Int. J. Mod. Phys. B 9, 1899 (1995).
${ }^{13}$ For a recent review, see Photonic Band Gap Materials, edited by C. M. Soukoulis (Kluwer, Dordrecht, 1996).

${ }^{14}$ D. R. Smith et al., J. Opt. Soc. Am. B 10, 314 (1993).

${ }^{15}$ W. Chen and D. L. Mills, Phys. Rev. Lett. 58, 160 (1987).

${ }^{16}$ C. Martine De Sterke and J. Sipe, in Progress in Optics, Vol. 33, edited by E. Wolf (Elsevier, Amsterdam, 1994).

${ }^{17}$ Qiming Li, C. T. Chan, K. M. Ho, and C. M. Soukoulis, Phys. Rev. B 53, 15577 (1996).

${ }^{18}$ E. Lidorikis, Qiming Li, and C. M. Soukoulis, Phys. Rev. B 54, 10249 (1996).

${ }^{19}$ R. P. Stanley, R. Houdre, U. Oesterle, M. Ilegems, and C. Weisbuch, Phys. Rev. A 48, 2246 (1993).

${ }^{20}$ P. F. Byrd and M. D. Friedman, Handbook of Elliptic Integrals for Engineers and Scientists (Springer, New York, 1971).

${ }^{21}$ K. Busch, C. T. Chan and C. M. Soukoulis, in Photonic Band Gap Materials, Vol. 315 of NATO Advanced Study Institute, Series E: Applied Science, edited by C. M. Soukoulis (Plenum, New York, 1996), p. 465. 